\title{
Mach keine Notizen, während Du mit Patienten sprichst
}

\begin{abstract}
Alle sechs Wochen trifft sich unsere Gruppe von zwölf Ärzten zur Supervision. Wir haben vor etlichen Jahren den Fähigkeitsausweis «psychosomatische und psychosoziale Medizin» erworben.

GF, Hausarzt in einem Dorf, berichtet über einen Patienten. Gewöhnlich spricht er frei. Diesmal aber hält er sich an die Krankengeschichte und zitiert fast wörtliche Einträge seines Gesprächs mit dem Patienten.

Unsere Gruppe bekundet Mühe, eine Gestalt des Patienten zu entwickeln, die somatische, psychische und soziale Gegebenheiten integriert. GF schildert, wie ihn beim Abholen des Patienten im Wartzimmer verunsichernde Gefühle beschlichen haben.

Wir vermuten, dass er dieses Mal die eigenen Gefühle nicht als mögliche Gegenübertragung [1] ins Gespräch mitnehmen konnte, sondern dass er mit fleissigem sofortigem Notieren seine Reaktion zu kontrollieren versuchte.
\end{abstract}

\section{Das Band}

«Wer will was Lebendigs erkennen und beschreiben,

Sucht erst den Geist herauszutreiben,

Dann hat er die Teile in seiner Hand,

Fehlt, leider! nur das geistige Band»

(Faust I, Mephistopheles zum Schüler).

Das hier gemeinte Band verbindet somatische, psychische und soziale Gegebenheiten. Dazu ein Beispiel aus dem Schachspiel: Einem Grossmeister wird für zwei Minuten ein Schachbrett gezeigt, auf dem die Partie beim zwanzigsten Zug steht. Dann wird das Brett abgedeckt und der Grossmeister hat die Partie auf einem Brett nachzustellen. Dies gelingt ihm rasch und korrekt dank seinem «geistigen Band». Wird ihm eine «Partie» gezeigt, bei der die Figuren nach Zufall aufgestellt sind, misslingt es.

Um das Band in der Anamnese zu verstehen, stellen wir uns den Organismus als Ebenen vor, die hierarchisch geschichtet sind, und vom Molekularen über die Zelle, den Zellverband, das Organ, die Organsysteme, das Unwusste, das Vorbewusste, das Bewusste, die Person, die Zweierbeziehung, die Familie bis in das Soziale reichen. Das Band verbindet auf einer Ebene horizontal deren Komponenten und vertikal die verschiedene Ebenen untereinander. Die hergestellten Verbindungen bezeichnen wir als durch Koppelungen gebildet, die Auflösung von Verbindungen nennen wir Entkoppelung [2]. Ein Beispiel: Vom Säuglingalter bis zum vierten Lebensjahr sind Magensäuresekretion und die Präsenz einer vertrauten Per- son gekoppelt. Im Verlauf des vierten Jahres entkoppelt sich diese Beziehung. Günstige Koppelungen erhalten die Gesundheit, pathologische führen zur Krankheit. Die Entkoppelung der Beziehung von Ehepartnern durch den Tod erhöht beim Hinterbliebenen zwei Jahre lang die Mortalität um $40 \%$ [3].

\section{Die Bedeutung der Anamnese}

$85 \%$ der für die Formulierung einer Diagnose nötigen Elemente stammen aus der Anamnese, der Rest aus Körperuntersuchung und Labordaten. Damit das Band in der Anamnese gefunden werden kann, müssen Arzt und Patient eine Beziehung eingehen, das Arbeitsbündnis, nach Greenson [4] die «working alliance». Es wird eine Situation gesucht, in der der Patient sich geborgen weiss und fühlt [5]. Im Buch «Anamnese und Körperuntersuchung» ist festgehalten, was der Arzt dazu beiträgt: Er stellt sich dem Patienten vor, achtet darauf, dass der Patient für das Gespräch optimal sitzt oder liegt, dass er nicht gestört wird, der Raum geschlossen ist, und der Patient sein momentanes Gefühl ausdrücken und seine Geschichte in seinen eigenen Worten, und ohne zur Unzeit unterbrochen zu werden, darlegen kann. Fragen sollen offen sein und keine Ja/Nein-Antworten anpeilen, Suggestivfragen und Doppelfragen sind zu vermeiden usw. Der Arzt soll sich in die individuelle Wirklichkeit [2] des Patienten versetzen, keine Notizen machen, und sich nur kurz ausklinken und Überlegungen anstellen. Sigmund Freud nannte das die frei schwebende Aufmerksamkeit, die nicht nur hinter der Couch angezeigt ist. Muss unbedingt geschrieben werden, bittet der Arzt den Patienten, einen Augenblick innezuhalten, notiert das Notwendige, und bittet dann den Patienten, fortzufahren.

\section{Patientenbeispiele}

Eine 22-jährige Studentin, die ich seit ihrer Kindheit kenne, bittet mich, sie zum neurologischen Konsilium zu begleiten. Es besteht Verdacht auf den ersten Schub einer multiplen Sklerose. Ich sitze in einer Ecke des Untersuchungszimmers, die Patientin sitzt nahe der Liege. Die Neurologin betritt das Zimmer, grüsst, setzt sich an den Computer und beginnt mit über ihre rechte Schulter gewendetem Kopf, Fragen zu stellen. Nach der Konsultation fühlt sich die Patientin unglücklich und ängstlich. Das In- 
terview hat keine psychischen und sozialen Daten erfasst. Seit 1970 vermutet man bei multipler Sklerose Zusammenhänge zwischen Ausbruch, Schüben und Belastungen [6].

Eine 74-jährige Frau wird wegen seit zehn Jahren anhaltenden Gesichtsschmerzen zugewiesen. In den vorhergehenden Jahren hat sie viele Ärzte aufgesucht. Im Einverständnis mit der Patientin benütze ich das Tonband. Das Interview ist im Wortlaut erfasst [7]. Sie ist froh, dass ich sie frage, ob sie Hörschwierigkeiten habe, und ich setze mich auf die Seite, auf der sie besser hört. Sie leide an fürchterlichen Schmerzen. Sie strahlen vom rechten Unterkiefer über den Nasenrücken in die Stirn, von dort in die Kopfhaut; Kämmen sei wegen Verstärkung der Schmerzen nicht mehr möglich. Ihr Mund sei furchtbar trocken, sie schlafe schlecht ... sie habe vor zehn Jahren einen ihrer Söhne verloren ... sie möchte nicht mehr leben und sie sei vergesslich ...

Nach 15 Minuten nehme ich den Faden beim verlorenen Sohn auf. Er sei mit dem Auto von der Strasse abgekommen, an einen Baum geprallt. Ich frage nach Einzelheiten: Hat er nach dem Unfall noch gelebt? Er sei sofort gestorben ... Sie erzählt, ein anderer Sohn habe gesagt «Gehe den Hans nicht schauen, behalte ihn im Gedächtnis so wie er vor dem Unfall war, sein Gesicht sei so sehr verunstaltet.» Dabei fährt sie mit ihrer Hand über ihr Gesicht, wie bei der Schilderung der eigenen Gesichtsschmerzen. «Drei Wochen später hat es angefangen ... das ist es!» ruft sie schluchzend [7]. Einige Gespräche mit der Patientin zu Trauer und Trauern führten zum Verschwinden der Schmerzen, und sieben Jahre später beim Followup berichtet sie, schmerzfrei geblieben zu sein. Zu Leidensbeginn sind die Ebenen der Familie und die des Körperteils Gesicht gekoppelt, durch die Trauerarbeit wurden sie entkoppelt.

\section{Gründe, die den Arzt zum Notieren während des Interviews bewegen}

Die Befürchtung, Wesentliches eines Gesprächs zu vergessen. Während der Ausbildung als Student und junger Arzt wird das Üben der Anamnese vernachlässigt. Erhält man Gelegenheit, zuerst zu erleben, wie viel Beobachtbares in einer 5-Minuten-Anamnese, später in einer zehnminütigen und noch später in einer längeren Anamnese enthalten ist, und darf man lernen, einfach zuzuhören und zu -sehen, findet man das integrierende Band und verliert die Angst, Wichtiges zu vergessen. Es erstaunt, wieviel man bei gleichschwebender Aufmerksamkeit behält. Ein Arzt, der keine so sorgfältige Einführung ins Erheben der Anamnese erlebt hat, kann sich später behelfen, indem er sei- ne Anamnese auf einen Tonträger aufnimmt und nach Abschluss des Gesprächs das Wichtige notiert und dann das Aufgenommene abhört und vergleicht, ob er Bedeutsames vergessen hat.

Das Zeitproblem: Eine Anamnese kann mehr als zwanzig Minuten beanspruchen. Wird aus Angst vor zu wenig Zeit hauptsächlich die System-Anamnese durchgeführt und darauf verzichtet, den Assoziationen des Patienten ins Psychische und Soziale zu folgen, dann spart man vermeintlich Zeit. Wie das Patientenbeispiel zeigt, muss man später mit viel mehr Zeitaufwand büssen.

Das Empathieproblem: Mit dem Teilnehmen an der Individuellen Wirklichkeit des Patienten wird man von dessen Gefühlen wie Wut, Angst, Trauer, Hilf- und Hoffnungslosigkeit erfasst. Mit Erfahrung lernt der Arzt, mitzufühlen, ohne in den Wirbel der Gefühle des Patienten hineingezogen zu werden, sodass er den Rückzug ins schützende Notieren weglassen kann.

Das juristische Problem: Notizen nach dem Gespräch schützen genauso gut vor einem juristischen Belangtwerden wie während des Gesprächs angefertigte Notizen.

Die Anamnese ist der Königsweg zum Band, das die Gegebenheiten aus den drei Bereichen Soma, Psyche und Soziales zusammenführt. Die Anamnese erfüllt die Forderungen des schweizerischen Gesundheitssystems: W-Z-W. Sie ist wirksam, zumutbar und wirtschaftlich.

\section{Bibliografie}

1. Gegenübertragung. In: Wörterbuch der Psychoanalyse; Frankfurt/M.: Suhrkamp: 1972

2. von Uexküll T, Wesiak W: Integrierte Medizin als Gesamtkonzept der Heilkunde: ein bio-psycho-soziales Modell. In: Adler RH, Herzog W, Joraschky P, et al. (Hrsg): Pychosomatische Medizin. Stuttgart: Urban \& Fischer: 2010.33-34.

3. Parkes CM: Bereavement. London; Tavistock: 1972.

4. Greenson RR: The technique and practice of psychoanalysis 1. NY: Internat. Univ. Press: 1967.

5. Adler RH, Hemmeler W: Anamnese und Körperuntersuchung. (3. Aufl.) Stuttgart: Fischer: 1992

6. Mei-Tal W, Meyerowitz S, Engel GL: The role of psychological process in somatic disorder: multiple sclerosis. 1. The emotional setting of illness and exacerbation. Psychosomatic Med 1970; 32:67-86.

7. Adler RH. Herausforderung für die Biomedizin: Das biopsychosoziale Konzept. Basel: EMH Media; 2017.

Prof. Dr. med. Rolf Adler

Leiserenweg 4

3122 Kehrsatz

michele.rolf.adler@gmail.com 